In Cres. Vol. $4 N^{\circ} 1:$ pp. 113-120, 2013

\title{
VIVENCIAS DE LA GESTANTE ADOLESCENTE EN LA PERSPECTIVA FENOMENOLÓGICA DE HEIDEGGER*
}

\author{
EXPERIENCES OF PREGNANT TEEN IN THE \\ PHENOMENOLOGICAL PERSPECTIVE \\ OF HEIDEGGER
}

\author{
Silvia Elizabet Reyes Narváez, \\ Carlos Alberto Tello Pompa ${ }^{2}$
}

\section{RESUMEN}

El presente artículo de aporte conceptual es uno de los productos de la investigación denominada: "Vivencias del embarazo en la gestante adolescente en una comunidad rural costeña", para optar el título de doctora en Ciencias de Enfermería; y tiene como propósito analizar comprehensivamente el ex-sistir femenino de la adolescente embarazada. Por esta razón el sentido del ser-ahí está dirigido a develar y comprender la vivencia del embarazo en la gestante adolescente en una comunidad rural costeña, de la provincia de Barranca.

El abordaje fenomenológico se basa en los conceptos de Martin Heidegger, filósofo con una decidida tendencia a lo existencial. Un elemento clave de su filosofía es la idea de lo sublime: aquello que trasciende la vida del hombre y le da sentido, lo sublime es la verdad y la verdad no es otra cosa que comprender el Ser.

El método fenomenológico heideggeriano propone la analítica existencial del ser-ahí a partir de la comprensión general del propio ser. La comprensión es la forma de penetrar en las posibilidades del ser, interpretándolo. Según Heidegger, esto significa percibir las posibilidades proyectadas por el Ser, las que son captadas por el sujeto investigador. En ese momento, las

* Recibido: 30 de marzo del 2013; aprobado: 18 de abril del 2013.

1 Maestra en Enfermería, mención: Salud de la mujer y el niño. Docente de la Universidad Nacional Santiago Antúnez de Mayolo - Filial Barranca, Perú.

2 Doctor en Enfermería. Universidad Federal do Rio de Janeiro. Docente de la Universidad Nacional de Trujillo, Perú. 
adolescentes embarazadas muestran lo que significa la gravidez, "su modo de ser" y su "siendo en el mundo estando embarazada", y nos permite conocer su "vivencia" sus formas de enfrentar una realidad inesperada y las posibilidades del fenómeno de ser madre adolescente.

La vivencia es una experiencia única que la joven madre vive física y emocionalmente en su mundo interior, y es la trayectoria fenomenológica la que nos permite comprender las experiencias de los sujetos en el proceso salud-enfermedad; y la que posibilita al enfermero(a) aproximarse más al ser humano a través de la comprensión de los fenómenos. De esta manera orienta la acción y transformación del cuidado al brindar nuevos elementos al cuidado científico y humano desde la perspectiva de la propia gestante adolescente.

PALABRAS ClAVE: Embarazo en adolescentes-vivencias, investigación cualitativa, fenomenología.

\section{ABSTRACT}

This article of onceptual contribution is one of the products of research entitled: "Experiences of pregnancy in pregnant teenager in a rural coastal community", to choose the title of Doctor of Science in Nursing, and is intended to comprehensively analyze the female ex-sist of pregnant adolescents. Thus, the sense of Dasein is aimed to reveal and understand the experience of pregnancy in the pregnant teenager in a rural coastal community of the province of Barranca (Peru).

The phenomenological approach is based on the concepts of Martin Heidegger, a philosopher with a strong tendency to the existential. A key element of his philosophy is the idea of the sublime: that which transcends human life and gives it meaning, the sublime is the truth and the truth is nothing to understand the Self.

According to Heidegger's phenomenological method proposes the existential analytic of Dasein from the general understanding of the self. Understanding is the way to penetrate the possibilities of being, interpreting. According to Heidegger, this means perceiving the possibilities projected by the Self, which are captured by the individual researcher. At that time, pregnant teens show what the pregnancy, "his way of being" and "being in the world while pregnant", and lets us know your "experience" their ways of coping with an unexpected reality and possibilities of phenomenon of teenage motherhood.

The experience is a unique experience that the young mother lives physicall and emotionally in his inner world, and is the phenomenological path that allows us to understand the experiences of individuals in the health-disease process, and that allows nurses (a) closer to the human being through the understanding of the phenomena. Thus, it guides action and transformation of care by providing new elements to the scientific and human care from the perspective of the very pregnant teenager.

KEY WORDS: Teenage pregnancy-experiences, qualitative research, phenomenology. 


\section{INTRODUCCIÓN}

La salud integral del adolescente debe ser entendida desde el punto de vista de su transcendencia, como un elemento básico para el desarrollo social del país y del mundo. En la población adolescente está no solo el futuro inmediato de una sociedad, sino también el presente, pues por sus características biopsicosociales propios de su edad, le permiten un mayor dinamismo, pero también una mayor exposición a múltiples riesgos, siendo uno de ellos el embarazo en la adolescencia. ${ }^{1}$

A nivel mundial, 14 millones de niñas adolescentes entre 15 y 19 años ya son madres. En Estados Unidos, 4 de cada 10 adolescentes se embaraza al menos una vez antes de cumplir los 20 años; en Chile, nacen, cada año, alrededor de 40355, cuyas madres son menores de 19 años; y estudios a pequeña escala en Brasil y Colombia indican que 1 de cada 10 mujeres hospitalizadas por aborto tiene menos de 20 años. ${ }^{2}$

En el Perú, según ENDES 2011, el 12,5\% de las adolescentes, entre 15 y 19 años, ya son madres o están embarazadas del primer hijo. La mayor prevalencia de embarazo se encuentra en el área rural $(19,7 \%)$ y las regiones de la selva $(25,3 \%)$; los datos también indican que el $16 \%$ de la mortalidad materna es en adolescentes. ${ }^{3}$

Existen muchas y muy variadas causas del embarazo en adolescentes, pero las más importantes son la falta de educación sexual, falta de madurez, carencia afectiva, inseguridad, baja autoestima, descuido por parte de los padres, falta de control de impulsos, violación, o simplemente curiosidad, consumo de alcohol y otros. Otro problema es la influencia cultural: los adolescentes se encuentran rodeados de material pornográfico, tanto en la televisión, como en el cine, la música, publicidad de cualquier producto, revistas, Internet y en los lugares de diversión y distracción, a los que los jóvenes asisten con frecuencia, cuyos mensajes "despiertan el interés por explorar lo desconocido"; muchas de las veces llegan a las relaciones sexuales porque "no tienen nada más que hacer". 1,4

El embarazo en las adolescentes es un problema de salud pública, y socialmente se le considera "la puerta de entrada al ciclo de la pobreza" y la feminización de la miseria. Estas jóvenes tienen mayor posibilidad de ser pobres, más probabilidad de divorcio, sus hijos con mayor riesgo de morbimortalidad y la alta proporción de ilegitimidad limita el acceso a los derechos legales y al cuidado de la salud. ${ }^{4}$ 
Según la Organización Mundial de la Salud (OMS), para cumplir con las metas de desarrollo en salud para el milenio, de la erradicación del hambre y la pobreza y de mejoramiento de la salud materna, se debe involucrar a todos los países y a todos los sectores sociales. El Perú prioriza estas metas en su Plan Concertado de Salud, con el que busca favorecer a las poblaciones más vulnerables, como la gestante adolescente. ${ }^{5}$

Si bien la maternidad está asociada a la adultez, no se pretende que la gestante adolescente, de la noche a la mañana, se convierta en adulta; ella sigue y seguirá por un tiempo siendo adolescente. Es cierto que en adelante la prioridad será el bebé por sobre los amigos, las fiestas y la moda, pero, es probable que ella todavía no lo haya aceptado. Se le debe ayudarla a tomar conciencia de los cuidados que requerirá en cuanto a su salud, sus controles, su alimentación y su conducta, de tal manera que su embarazo sea un acontecimiento feliz en su vida.

Una adolescente embarazada necesita del apoyo de sus padres, de su familia, de sus amigos y amigas para poder enfrentar la situación y no sentir que se le trunca la vida, sino que tiene un difícil reto por desafiar. Las instituciones y el estado también cumplen un rol importante en el apoyo y protección a la adolescente sea o no embarazada. Según el Plan Nacional de Acción por la Infancia y la Adolescencia 2002 - 2010, los adolescentes son agentes claves del desarrollo. En nuestro país, la Constitución Peruana y el Código de Niños y Adolescentes garantizan la protección de su salud física y emocional; de igual manera el Ministerio de Salud (MINSA) prevé la atención integral de la gestante y niño por nacer. ${ }^{6,7}$

Entre las soluciones que se plantean para disminuir o evitar el embarazo a temprana edad, es importante escuchar a la misma gestante adolescente, quien vive este proceso y es la más indicada para dar sugerencias. Por lo tanto, la comprensión de la vida cotidiana de la adolescente embarazada implica internarse en el lado sensible y práctico de esas relaciones sociales, de lo vivido y de lo concebido, de la subjetividad, singularidad, de las representaciones y de las imágenes, sin perder de vista, sin embargo, su criticidad, historicidad y contextualidad e identidad. $^{8}$

El hombre, como sujeto en el mundo, presenta vivencias de la existencia como cuidado, manifestación y comprensión; y en esa experiencia puede trascender a las condiciones de la existencia cotidiana, ya que el hombre además de ex-sistir para sí, también existe para los otros. De ese modo su existencia es inseparable de la existencia de los otros; ambas se entrelazan. Todos estos aspectos subsidian el cuidado en enfermería como un proceso inherente al proceso del cuidar y del existir y son expresados en las vivencias de las personas. ${ }^{9}$ 
El termino vivencia proviene del latín "vivere", que significa tener vida o existir. En este orden de ideas, la vivencia alude a la experiencia de vida, al modo de vivir. Husserl definía la vivencia como la propia experiencia que, con participación consciente o inconsciente del sujeto, se incorpora a su personalidad. De esta manera, las vivencias suponen las experiencias de lo que se vive en lo cotidiano de un sujeto en una determinada realidad siendo esta peculiar en su género. Vivencia es aquella experiencia que una persona vive en su vida y que de alguna manera entra a formar parte de su carácter. ${ }^{10}$

Para comprender como vivencia el embarazo de la adolescente se debe considerar su entorno familiar, historia de vida, relación de pareja, plan de vida, sentimientos y emociones, conflictos, contexto sociocultural; elementos que nos permiten entender su existencia cotidiana desde la perspectiva de la propia gestante.

Diferentes estudios reportan que el embarazo adolescente es un proceso que se experimenta en distintas formas según el entorno sociocultural analizado. Así lo demuestran los trabajos realizados por Ribeiro, Rojas y otros, quienes abordaron investigar diferentes aspectos de la vivencia del embarazo.

\section{ENFOQUE FENOMENOLÓGICO}

\section{ENFOQUE FENOMENOLÓGICO DE HEIDEGGER}

El referencial fenomenológico busca el significado de la experiencia vivida, contribuye a la búsqueda de la comprensión del hombre, sujeto de cuidado y viene, por lo tanto, al encuentro de los propósitos de la enfermería.

El concepto de fenomenología etimológicamente deriva del griego "Phainomenon", todo lo que se muestra, manifiesta o aparece ante un individuo que se interroga, y "logos", estudio o tratado. La fenomenología tiene su origen en el pensamiento de Edmund Husserl a inicios del siglo XX quien propugna que es un método que intenta entender de forma inmediata el mundo del hombre. ${ }^{11}$

La expresión fenomenología significa, primariamente, una concepción metodológica; no caracteriza el qué de los objetos de la investigación, sino el cómo de esta. La fenomenología es hacer ver desde sí mismo aquello que se muestra, y hacerlo ver tal como se muestra desde sí mismo. Heidegger define el concepto de fenomenología en "Ser y Tiempo", como lo que se muestra, sacar a la luz, hacer que algo sea visible en sí mismo, poner a la luz; es acercarse al mundo de la vida, a la vida del ser humano en el mundo. ${ }^{12}$ 
Los seres humanos tienen mundo. Para Heidegger estar en el mundo es exsistir, es estar involucrado, comprometido. Los seres humanos tienen un mundo que es diferente al ambiente, la naturaleza o el universo donde viven; es el todo, en el cual, los seres humanos se hallan inmersos en, y rodeados por. ${ }^{12}$

\section{VIVENCIAS DE LA GESTANTE ADOLESCENTE}

La adolescencia es una etapa importante en la vida, caracterizada por los cambios físicos y psicológicos, del enamoramiento y el inicio de las relaciones sexuales. El incremento de embarazo en adolescentes es preocupante, siendo necesario estudiar su mundo, cómo vive y siente este proceso marcado por sentimientos y emociones. El saber que está embarazada es una situación inesperada, algo que no estaba en sus planes, existiendo una ruptura entre su presente y su futuro. Su mundo interior está en conflicto, no sabe si siente alegría o tristeza, si decirle a sus padres o callarse, características propias de su etapa adolescente. Asimismo, su mundo exterior es de rechazo y de crítica, la sociedad aún es conservadora y no acepta a la joven madre al igual que su familia.

El embarazo en las adolescentes, en su mayoría, se inicia no muy auspiciosamente; su incursión en el mundo de ser madres es muy rápida. Perciben el embarazo como algo oscuro, árido y horrible, sienten amargura, tienen angustia porque están expuestas y sin apoyo de su entorno más cercano porque sienten el rechazo de sus padres. El sentirse embarazada es muy difícil para la adolescente, pues pasa de un momento a otro a desempeñar un rol para el cual no estaba preparada, inclusive sin haber cumplido su etapa como adolescente.

La joven madre es lanzada al mundo -ser propio lanzada al mundo- vive humanamente un embarazo que no esperaba, su ser-está-de caída. La gestante se muestra inauténtica como abandonada en el mundo y se pregunta ¿quién lo ha puesto en él? En ese momento, la mundaneidad, el ser mundo-con, les limita y fomenta una parte de la inautenticidad. En esa pugna, ellas no se sienten auténticas, no se sienten ser madres. Ese proceso les cuesta, pero tienen que encontrar una salida a pesar de su impotencia. El futuro es incierto; su ser-en-el-mundo se encuentra en un plano óntico, algo percibido y conocido como negativo y sin posibilidades; no se puede saber qué va a pasar, sin embargo, hay que afrontar esta situación de manera eficaz: ayudarla. El camino es largo y lleno de obstáculos, pero todos estamos involucrados en su cuidado, la familia, la sociedad y el estado. ${ }^{13}$

\section{EL SER EN LA BÚSQUEDA DEL CUIDADO DE SÍ}

Heidegger plantea que el cuidado, desde el punto de vista existencial, es 
ontológicamente anterior a toda actitud o situación del ser humano. El cuidado se encuentra en la raíz primera del hombre y representa un modo-de-ser esencial, presente, irreductible, constituyente y es base posibilitadora de la existencia humana. Habla del cuidado como anticipación, ocupación y solicitud, entendiendo que la noción de alteridad es intrínseca al mismo y que la expresión cuidado de sí sería una tautología. El cuidado surge cuando la existencia de otro adquiere importancia para sí misma; en consecuencia, se dispone a participar de su existencia; es ese modo-de-ser, mediante el cual, sale de sí misma para centrarse en el otro con desvelo y solicitud, desplaza la preocupación por sí misma y hace que el otro tome importancia para sí. ${ }^{12}$

En el escenario del cuidado también aparece el término pre-ocupación, que implica un adelantarse en el tiempo a los acontecimientos; sin embargo, la pre-ocupación no es un pensamiento negativo, aunque es cierto que genera intranquilidad y el ocuparse por adelantado de lo que va a suceder es una labor de los seres humanos en general y de la enfermería en particular. La preparación es precisamente uno de los elementos ontológicos que se generan a partir de la pre-ocupación; el ser humano se prepara para lo no acontecido, hace planes a fin de estar listo para el futuro. ${ }^{14}$

El comportamiento de sí mismo de las gestantes adolescentes es de cuidado, de "preocuparse de su vida" y de la "dedicación"; conciben el cuidado en un sentido ontológico y la percepción de las adolescentes es desafiar a la maternidad, pero necesitan fuentes de apoyo, de alguien con experiencia, que les brinde amor y comprensión; el apoyo de la familia, de la pareja y, en especial, de la madre, le permite hacer frente a esta nueva situación. Su futuro se percibe más abierto y con grandes posibilidades de asumir el rol de madre. Su ser propio está basado en la autenticidad, su decisión de aceptar el embarazo denota un cambio y es necesario el apoyo familiar.

\section{CONSIDERACIONES FINALES}

1. La fenomenología es un método que permite entender la existencia cotidiana y auténtica del proceso de embarazo en adolescentes.

2. Conocer la vivencia de la adolescente embarazada fortalece el área de conceptualización y práctica del cuidado, porque pone de manifiesto las descripciones de situaciones, eventos, interacciones y comportamientos; es decir cómo vive el embarazo, sentido y expresado por ellas mismas. 
3. Conocer y pensar sobre el ex-sistir de la mujer que vive un embarazo en la adolescencia permite tener una visión personal del mundo que se conoce.

\section{Estudiar a la gestante adolescente permite implementar, monitorear y} evaluar las políticas públicas, normas legales y declaraciones de protección a su salud física y psicosocial.

\section{REFERENCIAS BIBLIOGRÁFICAS}

1 ORgANIZACIÓN PANAMERICANA DE LA SALUD. OPS. 1999. Salud reproductiva en adolescentes. Estado Unidos: Volumen XVII $N^{\circ} 3$.

2 ORganizACIÓN MUNDIAL DE LA SALUd. OMS. 54a Asamblea mundial de la salud. Contribución de la OMS a la aplicación de la estrategia para la salud y el desarrollo del niño y del adolescente. 2009. [Internet]. Washington. Disponible en http://apps.who.int/gb/ebwha/pdf_files/WHA59/ A59_14-sp.pdf [Acceso 26 julio 2012].

3 ENCUESTA DEMOGRÁFICA Y DE SALUd FAMILIAR. Instituto Nacional de Estadística e Informática. 2011. [Internet]. Lima - Perú. http://desa.inei.gob.pe/endes/. [Acceso 4 de Febrero 2013].

4 ORGANIZACIÓN PANAMERICANA DE LA SALUD. OPS. La salud sexual y reproductiva del joven y del adolescente: oportunidades, enfoques y opciones. Washington, DC. 2008

5 ORgANiZACIÓN DE LAS NACIONES UnidAS. ONU. Objetivos del desarrollo del milenio en la ONU. 2008. [Internet]. Nueva York. Disponible en: http://www.un.org/spanish/ millenniumgoals/ [Acceso 4 de agosto 2012].

6 Plan Nacional de ACCIÓN POR LA INFANCIA Y LA AdOLESCENCIA 2002-2010. [Internet]. LimaPerú. Disponible en: http://www.oei.es/quipu/peru/PNAIA_2002_2010.pdf. [Acceso $10 \mathrm{de}$ agosto 2012].

7 MinISTERIO DE SALUD. Guía nacional de operativización del modelo de atención integral de salud. 2004. [Internet]. Lima-Perú. Disponible en: http://www.cimfweb.org/bn admin/include/images/ pdf/goapsperu.pdf. [Acceso 10 de agosto 2012].

8 TEDESCO, J. Educación y sociedad del conocimiento y de la información. Encuentro Internacional de Educación Media. Secretaría de Educación de Bogotá, Colombia, agosto de 1999. [Internet] Disponible en: http://www.iipe-buenosaires.org.ar/pdfs/bogota.pdf. [Acceso 24 de agosto 2012].

9 GaOs, J. Traductor de El ser y el tiempo de Heidegger. 3ed. México Madrid-Buenos Aires F. Cultura Económica. 2000.

10 Rocher, G. Introducción a la sociología general. 1ra edición. Herder-Barcelona. 2006.

11 LEÓN, A. El giro hermenéutico de la fenomenológica. En: Martín Heidegger. 2009. [Internet]. Disponible en:http://www.scielo.cl/pdf/polis/v8n22/art16.pdf. [Acceso 28 de agosto 2012].

12 HeIDegGer, M. El ser y el tiempo. Fondo de Cultura Económica, Madrid. 2000.

13 Inwood, M. Dicionário Heidegger. Rio de Janeiro: J. Zahar, 2002.

14 LÉVINAS, E. El tiempo y el otro. Barcelona: Paidós. 1993.

15 Ribeiro, PAtricia. Aun siendo un adolescente, soy una madre y me gusta ser de esta manera. Tesis Doctoral. Sao Paulo-Brasil. 2009. [Internet]. Disponible en: www.teses. Usp.br. [Acceso 24 de agosto 2012].

16 RoJAs, D; ALARCón, M; CALDERón, B. Vivencia del embarazo en adolescentes en una institución de protección en Colombia. [internet]. disponible en: http://dx.doi.org. [acceso 24 de octubre 2012]. reynaelizabet26@hotmail.com 